\title{
LENGUAJE, DISCRIMINACIÓN Y DISCAPACIDAD EN EL CONTEXTO EDUCATIVO DE MAGALLANES. UN ENFOQUE DE DERECHOS HUMANOS DESDE LA TERAPIA OCUPACIONAL
}

\author{
ANDREA YUPANQUI C. ${ }^{\alpha}$, MARÍA Á. GONZÁLEZ B. ${ }^{a}$, MIGUEL LLANCALAHUÉN V.a \\ WALTER QUILODRÁN O. ${ }^{a} \&$ CARLA TOLEDO A. ${ }^{a}$
}

\section{RESUMEN}

El presente artículo analiza la actual utilización de lenguaje discriminatorio hacia las personas con discapacidad, específicamente en el entorno escolar. De acuerdo al planteamiento de diversos autores (Echeverría, 1994; Maturana, 1998; Van Dijk, 1996, 2003; Van Dijk \& Bixio, 2009; Magendzo \& Donoso, 2000; Barton, 2005; Fairclough, 2010), a partir de la interacción comunicativa el lenguaje crea realidades, modela identidades y al mundo social, contribuyendo al abuso de poder y dominación. Ello abre un espectro de posibilidades discriminatorias hacia ciertas minorías sociales, lo que provocaría su segregación, modelo que también se reproduce al interior de las escuelas.

El objetivo de este estudio fue evaluar el impacto de un proceso de sensibilización y capacitación dirigido a comunidades educativas sobre la temática de la discapacidad y lenguaje inclusivo ${ }^{1}$. Su metodología fue mixta con un enfoque de Investigación Acción-Participativa, aplicado a cuatro establecimientos educativos de la ciudad de Punta Arenas, Chile, cuya muestra consideró a estudiantes, profesores y directivos. Se usaron técnicas de intervención comunitarias-participativas y sus técnicas de recolección de datos fueron entrevista semiestructurada y encuesta. Para el análisis de resultados se utilizaron procedimientos cualitativos de la Teoría Fundamentada de Glaser \& Strauss (1967), y el análisis cuantitativo se basó en una correlación estadística de tipo descriptiva.

En conclusión, se puede indicar que se utiliza en un alto porcentaje un lenguaje discriminatorio y no inclusivo en los establecimientos educacionales de la región, el cual repercute en las relaciones interpersonales y convivencia escolar, lo que como consecuencia, vulnera los derechos humanos de niñas, niños y jóvenes con discapacidad. La Terapia Ocupacional tiene la responsabilidad de eliminar las situaciones de apartheid ocupacional, promoviendo la justicia ocupacional en aquellos lugares donde exista discriminación en todas sus formas, mediante la modificación de barreras sociales, en virtud de construir una sociedad más inclusiva.

PALABRAS CLAVE: lenguaje, discriminación, discapacidad, derechos humanos, educación, terapia ocupacional.

a Universidad de Magallanes, Facultad Ciencias de la Salud, Departamento Terapia Ocupacional. Av. Bulnes Nº1855, Punta Arenas, Chile. andrea.yupanqui@umag.cl, mariadelosangeles.gonzalezb@gmail.com, miguel_llan7@hotmail.com, walter. quilodran@gmail.com, kartoledoa.to@hotmail.com 


\title{
LANGUAGE, DISCRIMINATION AND DISABILITY IN THE SCHOOL SETTING OF MAGELLAN EDUCATION. A HUMAN RIGHTS-BASED APPROACH FROM OCCUPATIONAL THERAPY
}

\begin{abstract}
This article puts emphasis on the discriminatory language used today to the person with disabilities, specifically in the school context. Several authors have examined how the communicative interaction, through the language, can creates realities, shapes identities and to the social world, contributing to the abuse of power and domination. It opens a spectrum of discriminatory possibilities towards certain social minorities, which would incite their segregation and those elements are reproduced through the spaces of the school (Echeverria, 1994; Maturana, 1998; Van Dijk, 1996; 2003; Van Dijk \& Bixio, 2009; Magendzo y Donoso, 2000; Barton, 2005; Fairclough, 2010).

The objective of this study was to evaluate the impact of a process of awareness and training aimed at educational institutions on the issue of disability and inclusive language. The study used a mixed methods (combining qualitative and quantitative methods research) with an approach of Participative-Action Research, applied to four educational establishments in the city of Punta Arenas, Chile, which sample considered students, teachers and authorities. Community participative techniques of intervention were used and the technical of compilation of information was semi-structured interview and survey. The results were analyzed using qualitative procedures of the Grounded Theory (Glaser \& Strauss, 1967), and the quantitative analysis on a statistical descriptive correlation.

In conclusion, this research indicates that a high percentage of discriminatory and noninclusive language is used in schools of the region, which affects interpersonal relationships and school life, thus violating the human rights of children and young with disabilities. Occupational Therapy has the responsibility of eliminating the situations of occupational apartheid, promoting the occupational justice in those places where discrimination exists in all forms, by the modification of social barriers, to constructing a more inclusive society.
\end{abstract}

KEY WORDS: language, discrimination, disability, human rights, education, occupational therapy.

\section{INTRODUCCIÓN}

El presente artículo aborda el uso del lenguaje como medio de discriminación hacia la población con discapacidad ${ }^{1}$. En el contexto nacional y bajo el marco de la Declaración Universal sobre los Derechos Humanos (ONU, 1948), el año 2008 Chile ratificó la Convención Internacional sobre los Derechos de las Personas con Discapacidad de las Naciones Unidas, primer tratado de Derechos Humanos del siglo XXI. Posteriormente, el 2010 entra en vigencia la Ley $\mathrm{N}^{\circ} 20.422$, la que establece Normas sobre

1 Proyecto patrocinado por el Programa EXPLORACONICYT Magallanes, Programa Nacional de Divulgación y Valoración de la Ciencia y la Tecnología, perteneciente
Igualdad de Oportunidades e Inclusión Social de Personas con Discapacidad (MIDEPLAN, 2010). Ambas normativas determinan que las personas no son discapacitadas sino que presentan una discapacidad y es el entorno el que las discapacita. Lo anterior refleja un cambio de paradigma en el tema de la discapacidad, considerándola como un tema de derechos humanos. Se afirma que la educación debe promover en las personas con discapacidad su participación en la sociedad, su sentido de dignidad, valor personal y el desarrollo de todo su potencial en lo que se refiere a la

a la Comisión Nacional de Investigación Científica y Tecnológica, organismo asesor dependiente del Ministerio de Educación del Gobierno de Chile. 
personalidad, talentos y creatividad (ONU, 2007), y por tanto, hoy es deber de cada Estado promover la igualdad de oportunidades de las personas con discapacidad

Los programas destinados a las personas con discapacidad que ejecute el Estado, deben tener como objetivo mejorar su calidad de vida, principalmente, a través de acciones de fortalecimiento o promoción de las relaciones interpersonales, desarrollo personal, autodeterminación, inclusión social y el ejercicio de sus derechos. De modo complementario, la Ley Chilena $N^{\circ} 20.536^{2}$, sobre Violencia Escolar del 2011, señala que cada establecimiento educacional debe promover la buena convivencia escolar y prevenir toda forma de violencia física o psicológica, agresiones u hostigamiento reiterado, realizado por estudiantes que atenten en contra de otro estudiante, valiéndose de una situación de superioridad o de indefensión de la víctima, que le provoque maltrato, humillación o temor fundado de verse expuesta a un mal de carácter grave (MINEDUC, 2011b). En definitiva, estas Declaraciones, Leyes y Convenciones han tenido el propósito de normar universalmente todo tipo de discriminaciones, poniendo un claro énfasis en la estrecha relación existente entre derechos humanos, convivencia entre las personas y nodiscriminación.

Antecedentes diversos demuestran que esta problemática se ha masificado tanto a nivel internacional como en nuestro país (FONADISINE, 2004; UNICEF, 2004; MINEDUCUNESCO, 2005, 2011; Miller et al. 1998; Duncan, 1999; Díaz-Aguado et al. 2013), y en esto, el discurso ha tenido un rol particularmente relevante como medio de discriminación, especialmente en su afectación a grupos minoritarios, e inevitablemente al originarse en nuestra sociedad se termina reproduciendo al interior de las escuelas. Revisiones sistemáticas han demostrado que niñas, niños y jóvenes con diversos tipos de discapacidad padecen altos niveles de bullying y victimización en contextos escolares, y es mayor con respecto a sus pares sin discapacidad (Mishna, 2003; Carter \&

2 Publicada en el Diario Oficial el 17 de septiembre de 2011, modifica la Ley General de Educación para tratar el tema de la violencia en los colegios y fijar las pautas
Spencer, 2006; Rose et al. 2011).

En consecuencia, el presente proyecto tuvo como objetivo evaluar el impacto de un proceso de sensibilización y capacitación dirigido a establecimientos educacionales sobre discapacidad y educación inclusiva en la ciudad de Punta Arenas, con el fin de analizar sus implicancias en futuras políticas educativas inclusivas.

\section{Lenguaje y discriminación}

La utilización del leguaje como un medio de discriminación, se explicaría por cuanto constituye un medio fundamental de comunicación, y a su vez, de reproducción principal de prejuicios e ideologías sociales en las cuales subyacen conceptos discriminatorios (Van Dijk, 2003, p. 79). El mismo autor correlaciona poder y discurso, donde pone en evidencia formas de dominación del discurso, discriminación comunicativa u otras formas de marginalización y exclusión que proceden de las estructuras del contexto y de las estructuras del discurso (Van Dijk, 1996, 2003; Van Dijk $\&$ Bixio, 2009). Lo anterior se alinea con los postulados de Echeverría (1994), quien afirma que el sistema de lenguaje es una estructura de relaciones y la posición de cada miembro de la comunidad dentro de esta estructura es un aspecto importante a considerar en el proceso de individualización (p. 59). En esto concuerda Fairclough (2010), quien reconoce que vivimos en una época lingüística, y enfatiza en las ideologías individuales, las que se presentan en forma implícita en la interacción lingüística de los sujetos y de las cuales, en general, no siempre se tiene plena conciencia (p. 10). Asî del contexto se integran prejuicios, matices despectivos, atribuciones arbitrarias. Aquí el impacto cultural del entorno queda demostrado mediante el uso del lenguaje, pues logra ubicar a las personas en situaciones de privilegio o marginación en la sociedad.

En particular en la temática de la discapacidad, sucede que muchas veces las

para que la comunidad escolar pueda abordar estas situaciones. 
personas con discapacidad son receptoras de respuestas ofensivas por parte de la sociedad (Barton, 2005, p. 24), y quizá la manera más obvia en que se manifiesta esta realidad es mediante la propagación de términos ofensivos empleados para descalificar, tratar como inferiores o insultar a individuos o grupos particulares por su condición de discapacidad (Islas, 2005, p. 7). Incluso la utilización de palabras por parte de la población, la mayoría de las veces es sin conciencia de su profundo e implícito poder $(O p$ cit, p. 5). Desde esta perspectiva, la utilización de términos como minusválido, inválido, incapacitado, lisiado, retrasado o subnormal, desde un enfoque lingüístico, corresponden a adjetivos calificativos, cuya función es promover la calificación o caracterización de sujetos, y en este caso, mediante connotaciones sociales despectivas, de inferioridad, de ineptitud, de dependencia. Por ejemplo, si atendemos al concepto minusválido, el significado de minus corresponde a menos, mientras que válido tiene referencia a valor. Es decir, con estos conceptos se expresa que dichas personas tendrían menos valor que el resto de la sociedad, o no tendrían valor (inválidos), o serían incapaces (incapacitados). En este sentido, resulta vital educar y crear conciencia sobre la relación entre lenguaje y poder, particularmente, sobre cómo el lenguaje contribuye a la dominación de unos y la opresión de otros (Fairclough, 2010, p. 3).

\section{Discapacidad y Discriminación: Conceptos}

El concepto actual de discapacidad atiende a un fenómeno complejo, el que refleja los aspectos negativos de la interacción entre personas con un problema de salud y las características de la sociedad en la que vive, tales como actitudes sociales negativas, transporte o edificios públicos inaccesibles o falta de apoyo social. Esta definición surge desde el nuevo paradigma de Derechos Humanos (en adelante $\mathrm{DDHH})$, vigente en la actualidad, desde el cual la dignidad, el pleno respeto de los DDHH y la igualdad de las personas determinan que la causa de la discapacidad radica predominantemente en los obstáculos que le impone el entorno. La concepción sobre discapacidad evoluciona desde un modelo biomédico a un modelo social (Barton,
2005; Blanco, 2004). Según la Clasificación Internacional de la Funcionalidad, esta definición también se complementaría con los déficits, limitaciones en la actividad y restricciones en la participación, motivo por el cual existiría un aspecto negativo en la interacción entre el individuo y los factores contextuales que lo involucran, con un fuerte énfasis en las barreras sociales como principales obstaculizadores (OMS, 2001, p. 30). Aquí el elemento restricciones en la participación coincide plenamente con la concepción de justicia ocupacional de Wilcock y Towsend (2007), quienes postulan que es el pilar del conocimiento de la Terapia Ocupacional como profesión, y se pone en riesgo cuando se reduce, se aliena, se margina, se explota o se devalúa de cualquier otra forma la participación de una persona. Su fase opuesta es denominada injusticia o apartheid ocupacional (p. 112).

La discriminación contra las personas con discapacidad alude a toda distinción, exclusión o restricción basada en una discapacidad (...) que tenga el efecto o propósito de impedir o anular el reconocimiento, goce o ejercicio por parte de las personas con discapacidad, de sus derechos humanos y libertades fundamentales (OEA, 1999, p. 20), y considerando lo anterior, para la Terapia Ocupacional, el elemento discriminación tendría plena relación con injusticia o apartheid ocupacional.

Discriminación y Discapacidad:

Un enfoque desde la Terapia Ocupacional

El abordaje de la discapacidad/discriminación y su prevención en todas sus formas ha sido una de las principales responsabilidades de la profesión de Terapia Ocupacional. En el ámbito educativo, su involucramiento ha sido progresivo desde el año 2005, impulsado por el Ministerio de Educación de Chile, entidad que plantea dentro de su Política Nacional de Educación Especial un fuerte compromiso por la promoción de una educación de calidad para todos, apoyando a los alumnos con mayores dificultades y a sus familias, como forma de hacer posible el derecho a la educación de todos sin exclusiones (MINEDUC, 2005, p. 46).

La profesión de Terapia Ocupacional se 
enfoca en la promoción de la salud y el bienestar a través de la ocupación en todos los ámbitos de desarrollo humano, en el que se incluye la educación. Su principal objetivo es promover el derecho de la participación activa de las personas en sus ocupaciones: la justicia ocupacional. Esto se logra a través de la habilitación de los individuos para realizar aquellas tareas que optimizarán su capacidad para participar o mediante la modificación del entorno para que refuerce la participación (WFOT, 2004) . $^{3}$

La Terapia Ocupacional es una disciplina que ha evolucionado con el propio desarrollo histórico de la humanidad, y la carrera en Magallanes no ha quedado excluida de este proceso. Estos últimos años la profesión se ha volcado hacia un enfoque social (Townsed \& Whiteford, 2007, p. 110), asumiendo su responsabilidad con la sociedad como una profesión promotora de un nuevo orden social, a favor de la superación de las desigualdades, la pobreza, las diferencias entre clases sociales y tender hacia el bien común, retomando un fuerte y marcado carácter humanista instaurado por sus fundadores (Clark \& Larson, 2001, p. 46). En este sentido, la formación de este profesional en valores sociales como la democracia, la humanización, la igualdad, fraternidad, y dignidad de los seres humanos, fomentando la justicia ocupacional, elementos que caracterizan la formación del Terapeuta Ocupacional en Magallanes. Por esto, su compromiso con la defensa de los derechos humanos en la sociedad ha ocupado un lugar prioritario estos últimos años, aplicado a todos los grupos etarios y en los diversos contextos en que se involucran los seres humanos.

En el contexto regional, resulta relevante abordar esta temática donde la profesión de Terapia Ocupacional se ha instalado hace 13 años en la Universidad de Magallanes, y su aporte a la comunidad educativa magallánica ha ido en franco aumento, desde 2008 hasta la fecha, cuando inicia un trabajo colaborativo con la Corporación Municipal de Punta Arenas y sus Proyectos de Integración Escolar. Desde entonces, el objetivo ha sido conformar equipos

3 WFOT corresponde a la Federación Mundial de Terapeutas Ocupacionales (World Federation of Occupational Therapists), organización oficial que representa a la Terapia Ocupacional a nivel internacional para su promoción desde el año 1952. El programa de Terapia Ocupacional de la Universidad de Magallanes recibió la acreditación internacional interdisciplinarios y apoyar la inclusión de alumnos con discapacidad a la educación regular, haciendo efectivo su derecho a la educación, igualdad de oportunidades y no discriminación. Por lo anterior, se ha considerado valioso generar esta estrategia de intervención en educación en valores y $\mathrm{DDHH}$ en los centros educativos, con el fin de aportar datos científicos sobre esta realidad local.

\section{Discapacidad y discriminación en cifras}

Estadísticas nacionales entregadas por el Primer Estudio Nacional de la Discapacidad del año 2004, muestran que un $12,9 \%$ de chilenos y chilenas vivían con discapacidad, lo que supone alrededor de 2.068.072 personas. En la región de Magallanes, sólo un 6,9\% de la población total presentaba algún tipo de discapacidad, lo cual indica que esta región se encuentra por debajo del promedio nacional (FONADIS-INE, 2004), pero que, igualmente refleja una realidad presente en Magallanes. El mismo estudio dio cuenta que 1 de cada 8 personas con discapacidad manifestaba haber sufrido algún tipo de violencia física o verbal, lo que correspondía a 274.599 personas.

De este modo también ocurre con la población en etapa escolar, demostrado por la UNICEF ${ }^{4}$ el año 2004, en un estudio sobre la convivencia escolar, donde se evidencia que en Chile, el $31 \%$ de niños, niñas y adolescentes, entre 12 y 18 años, incluso sin discapacidad, se han sentido discriminados en su escuela. Las formas más mencionadas se relacionaban con bromas descalificatorias por defectos físicos (UNICEF, 2004). El año 2005 se demuestra que alrededor de un tercio de los alumnos considera que con frecuencia es insultado o agredido verbalmente por compañeros (MINEDUC-UNESCO, 2005). Luego, el año 2011, la Encuesta Nacional de Prevención, Agresión y Acoso Escolar aplicada a los estudiantes de $8^{\circ}$ año básico, afirma que 1 de cada 5 estudiantes manifiesta que las amenazas $\mathrm{u}$ hostigamientos entre sus compañeros son habituales. De todos los tipos de agresión, lo más frecuente en

de la WFOT por 7 años, desde 2010 a 2017 y reacreditación nacional de Akredita por 4 años (2014-2018).

4 El Fondo de las Naciones Unidas para la Infancia o Unicef, fundado en 1946, es un programa de la Organización de las Naciones Unidas que provee ayuda humanitaria y de desarrollo a niños y madres en países en desarrollo. 
las escuelas son los insultos y burlas, con un $69 \%$ todos los días, varias veces a la semana o al mes. En consecuencia, la conducta de maltrato verbal es la conducta que afecta a un mayor porcentaje de estudiantes (MINEDUC, 2011a).

A nivel mundial los estudios vinculados al tema comenzaron en los países nórdicos, y con ellos la implementación de programas para disminuir las situaciones de hostigamiento. Posteriormente, el estudio de este fenómeno se extendió a otros países europeos y a Estados Unidos (Milicic \& López, 2008, p. 4). En la década de los ' 90 , se muestra que el $68 \%$ de los niños en edad escolar han sido hostigados, al menos, en dos ocasiones (Miller et al. 1998). Otros hablan de que un $97 \%$ de las víctimas de hostigamiento sufrieron ataques verbales, el $40 \%$ una o dos veces a la semana, el $43 \%$ tres o más veces a la semana (Duncan, 1999). Otro estudio realizado en Perú el año 2009, indica que la población con discapacidad o defecto físico se encuentra más expuesta a la violencia escolar severa (Amemiya et al. 2009). Así también un trabajo sobre acoso adolescente en España revela que el tener alguna discapacidad es reconocida en un $12,79 \%$ como una característica de las víctimas a las que los acosadores atribuyen la agresión (DíazAguado et al. 2013, p. 370).

Lenguaje Inclusivo:

Una dimensión del reconocimiento de los Derechos Humanos en la discapacidad

El lenguaje ocupa un lugar trascendental en la convivencia entre los seres humanos. Al respecto, Maturana (1998), sostiene que el lenguaje es mucho más importante para la convivencia de lo se había creído hasta ahora, es mucho más que un sistema de simbolos para comunicarnos, tiene que ver con las emociones y que ellas también son decisivas para la convivencia humana (p. 3).

Una respuesta desde la perspectiva de los derechos humanos a esta problemática es el uso del lenguaje inclusivo, un lenguaje libre de discriminación. Así como las diferencias entre hombres y mujeres requieren de un lenguaje inclusivo, también es

5 Esta metodología se enmarca en el desarrollo de las ciencias sociales de 1960, para explicar las relaciones entre el conocimiento, la acción y la transformación social. Se propuso como una alternativa para conocer los contextos comunitarios desde la acción, con desarrollos teóricos y metodológicos alternativos necesario al referirse a personas con discapacidad, o a quienes viven en situación de pobreza, donde el acento se debe poner en ser persona, y no en sus carencias o limitaciones. Esta forma resalta una condición positiva, y permite avanzar hacia un trato de igualdad (INDH, 2012, p. 37). Desde el marco de la convivencia social democrática y de la cultura inclusiva de derechos humanos, hoy se hace imprescindible promover la valoración de la diferencia y diversidad, reconociendo su indiscutible aporte a la sociedad. En el caso de la discapacidad, la forma inclusiva corresponde a persona con discapacidad, resaltando y valorando en primer lugar el concepto persona, valorando al sujeto, respetando su rasgo distintivo, el de persona en primera instancia, quien posee múltiples características, roles, intereses, necesidades, con capacidades y limitaciones, dando lugar una concepción de respeto y aceptación de diversidad para todo ser humano sin distinción. Esto marca la eliminación de una barrera social mediante el lenguaje, favoreciendo su reconocimiento integral como ser humano. Cuando el lenguaje hace estas distinciones, contribuye fehacientemente a un verdadero cambio cultural, extinguiendo mitos, prejuicios y estereotipos que atentan contra una sana convivencia en la diversidad.

En sintesis, resulta trascendental integrar una visión de respeto de derechos humanos de las personas con discapacidad, con una utilización de lenguaje que promueva valores de respeto, tolerancia, fraternidad, con el uso inclusivo del lenguaje, lo que sólo puede alcanzarse en una sociedad que posea una cultura verdaderamente inspirada en el reconocimiento irrestricto de los derechos que son esenciales para el ser humano (Comisión Nacional de Verdad y Reconciliación, 1996, p. 1290). Instalar esta temática en los contextos educativos es el desafío que hoy se hace urgente.

\section{MÉTODO}

La metodología utilizada en este estudio fue mixta (cuali-cuantitativa) con un enfoque de Investigación Acción-Participativa (IAP $\left.{ }^{5}\right)$. La IAP

frente a la rigidez de las investigaciones tradicionales. Su propuesta se fundamenta en la crítica a la investigación clásica, por su carácter descriptivo, neutral, teoricista, estadístico (Cifuentes, 2011, p. 57). 
es una metodología para diagnosticar, intervenir y evaluar los procesos psicosociales y que, principalmente, enfatiza en el protagonismo de los participantes (Krausse, 2002, p. 48). En la IAP las mismas personas investigan la realidad para transformarla. Se constituye en un paradigma de la investigación social, cuyo objetivo en el ámbito educativo es mejorar la práctica pedagógica, más que generar conocimientos (Elliot, 1996, p. 67).

Una de las principales características de la IAP, es empoderar a las personas y hacerlas conscientes de las problemáticas sociales que las afectan. Su gran potencial radica en la producción de conocimiento, articulando de manera crítica los aportes de la ciencia y del saber popular, con el fin de reorientarlos hacia la acción transformadora de la realidad, desencadenando intercambios constructivos entre investigador y comunidad en los que se abordan conjuntamente todas las etapas del proceso investigativo y de intervención social. En su conjunto, estas características fundamentan su utilización para para este estudio, pues permite a los integrantes de las comunidades educativas la sensibilización con respecto a la discriminación hacia las personas con discapacidad, junto con la búsqueda de soluciones a través de la participación activa de los actores involucrados, con el fin de promover un entorno inclusivo en los establecimientos educativos intervenidos.

\section{Descripción Proyecto IAP en Lenguaje,} Discapacidad y Derechos Humanos

El proyecto general fue dirigido por una académica del Departamento de Terapia Ocupacional de la Universidad de Magallanes, y la asesoría metodológica a cargo de una académica de la Dirección de Investigación de la Universidad de Magallanes. Dicho proyecto se enmarcó en línea de desarrollo de la unidad académica de ciencia de la ocupación y derechos humanos, patentado en la misión del Departamento: la búsqueda permanente de la ética y la justicia ocupacional (UMAG, 2015)

Este proyecto fue postulado, aceptado y patrocinado por EXPLORA-CONICYT Magallanes. En su ejecución participaron activamente 4 alumnos

6 El Programa de Integración Escolar una estrategia del sistema escolar chileno, que tiene el propósito de contribuir en el mejoramiento continuo de la calidad de la educación tesistas de la Carrera de Terapia Ocupacional de la Universidad de Magallanes, quienes trabajaron durante los meses de Mayo a Diciembre de 2012 con los integrantes de las comunidades educativas de la región. Su objetivo general fue evaluar el impacto de un proceso de sensibilización y capacitación dirigido a establecimientos educacionales sobre la temática de discapacidad y educación inclusiva en la ciudad de Punta Arenas. Los objetivos específicos fueron:

- explorar y describir el lenguaje vinculado a discapacidad que utilizan los miembros de las comunidades educativas;

- conocer las propias concepciones atribuidas a discapacidad y discriminación por parte de actores vinculados a la intervención;

- conocer la percepción de los miembros de las comunidades educativas en relación a su evaluación del proceso de sensibilización y capacitación vivido;

- conocer la percepción de los miembros de las comunidades educativas en relación a la valoración de aprendizajes y el impacto en la convivencia escolar producida por su participación en la intervención.

\section{Procedimiento}

Se contactó a diversos establecimientos y se les solicitó autorización a las autoridades de distintas comunidades educativas, explicando los objetivos del proyecto. Los establecimientos fueron seleccionados por los criterios de heterogeneidad, disponibilidad y por aceptar voluntariamente participar en el proyecto. Las 4 instituciones seleccionadas reflejan parte de la diversidad de realidades escolares en la región. Estas fueron:

- Establecimiento particular subvencionado sin Programa de Integración Escolar (PIE) ${ }^{6}$, con enseñanza desde preescolar a cuarto año medio; distribuidos de manera mixta (hombres y mujeres).

- Establecimiento municipal con PIE, con enseñanza desde preescolar a octavos años básicos; organizados de manera mixta.

que se imparte en el establecimiento, favoreciendo el ingreso y apoyo a estudiantes con diversos tipos de discapacidades (MINEDUC, 2012). 
- Establecimiento municipal con PIE, ubicada en zona rural. Cuenta con enseñanza desde preescolar a sexto básico, distribuidos de manera mixta.

- Establecimiento de educación especial, municipalizado, dirigido a niños, niñas y adolescentes, los que presentan en su mayoría discapacidad intelectual con distintos grados de severidad.

En cada una de estas instituciones se conformaron grupos de trabajo en cada establecimiento, conformados por 10 alumnos y 1 profesor. La selección de estos participantes fue probabilística de tipo intencional, procedimiento utilizado para priorizar la facilidad de acceso a estudiantes y profesor según las condiciones impuestas por cada institución. Se contactó al adulto responsable del niño, niña o joven y se les invitó a participar del estudio.

De esta manera, se diseñó un procedimiento que combinó la investigación con la intervención. Dicho procedimiento implicó la evaluación mixta, tanto de procesos como de resultados. Se ejecutó en 5 etapas:

\section{Evaluación Inicial}

La realización de una evaluación diagnóstica dirigida al grupo de trabajo sobre el uso de lenguaje inclusivo/no-inclusivo. Luego, cada grupo de trabajo conformado aplicó el mismo instrumento diagnóstico en su respectiva comunidad educativa. Evaluación cuantitativa.

\section{Intervención:}

Cada grupo participa de un taller teóricopráctico sobre la temática estudiada, a cargo de estudiantes tesistas de la Universidad de Magallanes. Los temas específicos tratados en los talleres fueron:

- Discapacidad, uso de lenguaje inclusivo y derechos humanos

- Bullying y sus consecuencias

- Inclusión y exclusión de personas con discapacidad.

- Reflexión y análisis sobre hallazgos encontrados en el uso de lenguaje inclusivo/noinclusivo en sus comunidades educativas

- Preparación de presentaciones para Con- greso Explora-Conicyt

III. Evaluación de Proceso:

Se buscó conocer y describir el cambio producido en el uso del lenguaje vinculado discapacidad en los grupos intervenidos. Evaluación cuantitativa.

\section{Difusión:}

Cada equipo de trabajo, junto a su profesor guía, presentan los resultados de su investigación en el Congreso Regional Escolar de Ciencia y Tecnología Explora-Conicyt, donde alumnos y profesores demostraron conocimientos adquiridos, valoración de los aprendizajes obtenidos. Presentaciones en modalidad oral y poster.

\section{Evaluación Final:}

Se intentó conocer y describir los resultados del proceso de investigación-acción, en términos de la valoración de aprendizajes y de su impacto en la convivencia escolar de su realidad local. Evaluación cualitativa.

\section{EVALUACIÓN CUANTITATIVA}

\section{Participantes}

1) Grupos de trabajo por establecimiento educativo

Los grupos de trabajo quedaron conformados en total por 40 estudiantes y 4 profesores. Sus características se detallan en la Tabla 1.

\section{2) Evaluación diagnóstica de comunidades educativas}

Se estableció un universo de 736 personas pertenecientes a los miembros de los cuatro establecimientos seleccionados de la ciudad de Punta Arenas. Las unidades de observación fueron profesores, directivos, estudiantes de primer, segundo y tercer ciclo. El tamaño definitivo de la muestra fue de 598 encuestados, con un margen de error del $3 \%$ y un nivel de confianza de $99 \%$. Se optó por una modalidad de muestreo no probabilístico de tipo intencional, favoreciendo la participación voluntaria de los sujetos en la investigación. La Tabla 2 indica el tamaño muestral y su distribución según cada establecimiento. 
Tabla 1. Caracterización de los grupos de trabajo.

\begin{tabular}{llll}
\hline Tipo de establecimiento & Edades estudiantes & Curso & Profesor guía \\
\hline Particular Subvencionado sin PIE & $14-15$ años & $1^{\circ}$ medio & Orientadora \\
Municipal con PIE - Urbano & 10 años & $5^{\circ}$ básico & Profesora de historia \\
Municipal con PIE - Rural & $9-10$ años & $4^{\circ}$ básico & Profesora educación básica \\
Municipal - Escuela Especial & $13-16$ años & Nivel 9 & Educadora diferencial \\
\hline
\end{tabular}

Fuente: Autores

Tabla 2. Caracterización de la muestra para el diagnóstico de comunidades educativas.

\begin{tabular}{|c|c|c|c|c|c|}
\hline $\begin{array}{l}\text { Tipo de } \\
\text { Establecimiento }\end{array}$ & $\begin{array}{c}\mathrm{N}^{\circ} \text { profesores } \\
\text { y directivos }\end{array}$ & $\begin{array}{c}\mathrm{N}^{\circ} \text { alumnos } \\
\text { primer ciclo } \\
3^{\circ} \text { y } 4^{\circ} \text { año } \\
\text { básico }\end{array}$ & $\begin{array}{c}\mathrm{N}^{\circ} \text { alumnos } \\
\text { segundo ciclo } \\
5^{\circ} \text { a } 8^{\circ} \text { año } \\
\text { básico }\end{array}$ & $\begin{array}{c}\mathrm{N}^{\circ} \text { alumnos } \\
\text { tercer ciclo } \\
1^{\circ} \text { a } 4^{\circ} \text { año } \\
\text { medio }\end{array}$ & $\begin{array}{c}\text { Total } \\
\text { Muestra } \\
\mathrm{n}\end{array}$ \\
\hline Particular Subvencionado sin PIE & 25 & - & - & 268 & 293 \\
\hline Municipal con PIE - Urbano & - & - & 241 & - & 241 \\
\hline Municipal con PIE - Rural & 6 & 28 & 17 & - & 51 \\
\hline Municipal - Escuela Especial & 13 & - & - & - & 13 \\
\hline Total muestra & 44 & 28 & 258 & 268 & 598 \\
\hline
\end{tabular}

Fuente: Autores

Técnica de recolección de datos cuantitativos

Para la exploración y descripción del lenguaje utilizado por la comunidad educativa, durante el primer semestre del 2012, se diseñó una encuesta basada en el Manual de recomendaciones para el uso correcto del lenguaje en temas relacionados con Discapacidad del Servicio Nacional de la Discapacidad (SENADIS, 2010). Su objetivo fue identificar la utilización de terminología sobre discapacidad por la muestra seleccionada. El equipo optó por un diseño simple, con el fin de uniformar el instrumento y poder ser aplicado por todos los estudiantes de los establecimientos educativos intervenidos y para edades diversas. Este instrumento se construyó con una sola sección, la que consultaba respecto de 30 conceptos vinculados a la temática de discapacidad e indagaba sobre su utilización por parte de los encuestados. Ellos debían responder marcando una cruz los conceptos que utilizan frecuentemente o han utilizado para referirse a la discapacidad.

La primera versión preliminar de la encuesta se aplicó a estudiantes dentro de la Universidad de Magallanes. A los estudiantes se les solicitó responder la encuesta, y posteriormente, se discutieron aspectos críticos en cuanto a la validez de constructo, la capacidad autoexplicativa de los conceptos y la claridad del formato para responder. Ese proceso fue esencial para evaluar la pertinencia del instrumento. A partir de sus comentarios, se validaron los conceptos consultados y se ajustó el lenguaje utilizado de manera que facilitara la claridad en la comunicación con los estudiantes encuestadores y con los encuestados. Para finalizar la validación del instrumento fue revisado por un metodólogo experto. Los conceptos consultados fueron:

- 9 TÉRMINOS INCLUSIVOS: Persona con discapacidad, persona con discapacidad física, persona con discapacidad intelectual, persona con discapacidad visual, persona con deficiencia visual, persona con esquizofrenia, persona con discapacidad auditiva, persona que usa silla de ruedas, persona sorda.

- 21 TÉRMINOS NO INCLUSIVOS: Insano, demente, mongolito, ciego, lisiado, inválido, paralítico, retrasado, loco, trastornado, in- 
vidente, cieguito, anormal, esquizofrénico, discapacitado, mongólico, incapacitado, sordomudo, enfermito, minusválido, retardado.

Estas encuestas se aplicaron a las cuatro comunidades educativas de los establecimientos durante los meses de agosto y septiembre de 2012, y su duración osciló entre 15 y 20 minutos. Cada estudiante, con supervisión y apoyo del profesor guía, encuestaba a las personas que tenía mayor acceso durante su permanencia diaria en el establecimiento educativo.

\section{Técnica de análisis datos cuantitativos}

El análisis de los datos cuantitativos se basó en una correlación estadística de tipo descriptiva.

\section{EVALUACIÓN CUALITATIVA}

\section{Participantes:}

Como procedimiento de muestreo se optó por uno de tipo no probabilístico de tipo intencional, de acuerdo a criterios de acceso y aceptación voluntaria. La Tabla 3 presenta las características de las unidades de observación, quienes representaron las distintas perspectivas de las personas que componen las comunidades educativas.

Tabla 3. Caracterización de la muestra para el análisis cualitativo

\begin{tabular}{cll}
\hline Informante & \multicolumn{1}{c}{ Rol } & Género \\
\hline 1 & Estudiante & Mujer \\
2 & Estudiante & Hombre \\
3 & Profesor- Cargo directivo & Mujer \\
4 & Profesor & Hombre \\
5 & Profesor & Mujer \\
6 & Profesor- Cargo directivo & Mujer \\
\hline
\end{tabular}

Fuente: Autores

Técnica de recolección de datos cualitativa

Se diseñó una entrevista individual semiestructurada, con el propósito de conocer y describir la perspectiva de las personas que participaron de la intervención y autoridades de algunos establecimientos educativos. La validación de esta entrevista fue realizada por dos muestras pilotos con similitud de género, rango etario y nivel educacional de acuerdo a los informantes reales, quienes dieron su apreciación y las posibles modificaciones al respecto. Además, el término de la validación lo dio un metodólogo experto, siendo este paso fundamental para evaluar la pertinencia del instrumento.

Para la elaboración de la entrevista se plantearon 4 ejes temáticos referidos a los temas principales vinculados a la intervención realizada:

- Concepto personal y social atribuido a discapacidad.

- Visión sobre la discriminación hacia las personas con discapacidad.

- Descripción del lenguaje personal y del utilizado por su comunidad educativa para referirse a las personas con discapacidad.

- Respecto de la intervención realizada. Evaluación, valoración e impacto.

Las entrevistas fueron grabadas y transcritas para su posterior análisis.

Técnica de análisis datos cualitativos

El análisis de la información recopilada a través de las entrevistas toma como eje los procedimientos consignados por la Teoría Fundamentada (Glaser \& Strauss, 1967; Strauss \& Corbin, 2002), abarcando sus tres etapas: codificación abierta, axial y selectiva.

\section{Aspectos éticos de la investigación}

- Participantes estudiantes menores de 12 años y jóvenes con discapacidad intelectual:

Se contactó a los adultos (padres y/o representantes legales) y se les describe la investigación, sus objetivos y características, se explicó sobre riesgos y beneficios, derecho a su participación libre, confidencialidad, el contacto con los responsables de la investigación y la explicitación de la voluntariedad de ingresar y continuar en el estudio. Quienes aceptaron, firmaron un consentimiento informado que autorizaba la 
participación del hijo o hija en el estudio. Luego se contactó a los niños, niñas y jóvenes, a quienes se les explicó la investigación. Ellos accedían a participar a través de un asentimiento informado que se les leía, y en el cual consignaban libre y voluntariamente que aceptaban realizar la actividad, con el derecho a su participación libre, pudiendo negarse o interrumpir su participación en cualquier momento. En todos los casos se resguardó el anonimato de los participantes y se aseguró que los hallazgos serían utilizados sólo con fines de la investigación.

- Participantes adolescentes (entre 12 y 18 años) y adultos:

El procedimiento de consentimiento informado se aplica en forma individual, respetando los mismos principios éticos de la investigación planteados anteriormente.

\section{RESULTADOS}

A continuación se presentan los resultados obtenidos de acuerdo a los tipos de análisis descritos para abordar este proyecto.

\section{Análisis cuantitativo}

Evaluación diagnóstica sobre el lenguaje utilizado por integrantes de las comunidades educativas

Se encontró que los conceptos más utilizados son los no inclusivos para referirse a las personas con discapacidad, con una significativa diferencia (más de 40\%) entre el concepto no inclusivo y el inclusivo más usado, lo que refleja la mayor tendencia por el uso de terminología no inclusiva en los miembros de las comunidades educativas. En la Tabla 4 se presenta la distribución de frecuencias y porcentajes de los conceptos discriminadores y no inclusivos más usados por la población encuestada, donde destaca que más de la mitad de los encuestados manifiesta usar los términos discapacitado $(58,4 \%)$ y ciego $(57,5 \%)$. Un alto porcentaje también lo obtuvieron los conceptos loco $(48,8 \%)$ inválido $(46,8 \%)$ y sordomudo $(43,8 \%)$.

En ambas tablas, el número total de personas que respondieron fue $\mathrm{n}=598$, y corresponde al valor a partir del cual se calcularon los porcentajes de respuesta, considerando que la consulta es multirrespuesta.

Tabla 4. Utilización de conceptos no inclusivos sobre discapacidad en comunidades educativas.

\begin{tabular}{lcc}
\hline Conceptos no inclusivos & $\begin{array}{c}\text { Frecuencia de } \\
\text { respuestas }\end{array}$ & Porcentaje \\
\hline Discapacitado & 349 & 58,4 \\
Ciego & 344 & 57,5 \\
Loco & 292 & 48,8 \\
Inválido & 280 & 46,8 \\
Sordomudo & 262 & 43,8 \\
Enfermito & 216 & 36,1 \\
Cieguito & 201 & 33,6 \\
Minusválido & 174 & 29,1 \\
Esquizofrénico & 151 & 25,3 \\
Retrasado & 143 & 23,9 \\
Anormal & 134 & 22,4 \\
Trastornado & 99 & 16,6 \\
Demente & 94 & 15,7 \\
Mongólico & 86 & 14,4 \\
Paralítico & 85 & 14,2 \\
Incapacitado & 81 & 13,5 \\
Retardado & 72 & 12,0 \\
Invidente & 47 & 7,9 \\
Lisiado & 46 & 7,7 \\
Insano & 31 & 5,2 \\
Mongolito & 26 & 4,3 \\
\hline Fuente Autes & &
\end{tabular}

Fuente: Autores

La Tabla 5 presenta la distribución de frecuencias y porcentajes de los conceptos inclusivos más utilizados, donde los valores más altos corresponden a los términos persona que usa silla de ruedas $(12,9 \%)$, persona con discapacidad $(11,5 \%)$ y persona sorda $(10,5 \%)$.

\section{Implementación de estrategia}

educativa en establecimientos

Se buscó conocer y describir el cambio producido en el uso del lenguaje vinculado a discapacidad mediante la aplicación de la encuesta. 
Tabla 5. Utilización de conceptos inclusivos sobre discapacidad en comunidades educativas.

\begin{tabular}{lll}
\hline Conceptos Inclusivos & $\begin{array}{l}\text { Frecuencia de } \\
\text { respuestas }\end{array}$ & Porcentaje \\
\hline $\begin{array}{l}\text { Persona que usa silla de } \\
\text { ruedas }\end{array}$ & 77 & 12,9 \\
$\begin{array}{l}\text { Persona con discapacidad } \\
\text { Persona sorda }\end{array}$ & 69 & 11,5 \\
$\begin{array}{l}\text { Persona con discapacidad } \\
\text { física }\end{array}$ & 49 & 10,5 \\
$\begin{array}{l}\text { Persona con discapacidad } \\
\text { auditiva }\end{array}$ & 42 & 8,2 \\
$\begin{array}{l}\text { Persona con discapacidad } \\
\text { intelectual }\end{array}$ & 32 & 7,0 \\
$\begin{array}{l}\text { Persona con discapacidad } \\
\text { visual }\end{array}$ & 21 & 5,4 \\
Persona con esquizofrenia & 17 & 3,5 \\
\hline
\end{tabular}

Fuente: Autores

En la Tabla 6 es posible observar cómo la intervención realizada tuvo un impacto positivo en la comprensión de la temática tratada, demostrado en el aumento de términos inclusivos y disminución de no inclusivos.

\section{Análisis cualitativo}

Eje temático I:

Concepto personal y social

atribuido a discapacidad

El concepto personal de discapacidad, releva la dualidad capacidad-discapacidad. Se hace un reconocimiento de lo que separa a los individuos entre ellos y los clasifica en categorías. Se menciona que estas personas no han desarrollado capacidades o bien, poseen capacidades que son diferentes.

Lo que significa para mí después de obviamente haberme informado bastante, es que una persona en alguna de las capacidades que todos tenemos, una de esas él no las tiene desarrolladas (E3, p. 1).

Si lo vemos en lo social, la gente tiende a pensar que la persona con discapacidad es alguien que tiene que estar como... mirarlo como como algo extraño, que está en una esquina, en un lugar que debemos aceptar que no puede hacer ciertas cosas y que también nosotros tenemos que ayudarlo (E4, p. 55).

El concepto que la sociedad actual tiene sobre la discapacidad según los entrevistados, corresponde a una visión que no valida o incluso anula a estas personas como tales en la sociedad, esto se debe a un concepto que considera a las personas con discapacidad como algo extraño o distinto, que necesitan ayuda o más atención por parte de toda la sociedad.

Lo que pasa es que hemos necesitado quizás utilizar esta palabra discapacidad para llamar la atención... en relación a lo que significa vivir con un otro o una otra que requiere quizás de más ayuda o de más atención en algunos casos... para que esa persona también tenga un lugar que sea amable para vivir y convivir (E6, p. 6).

Los resultados indican que el uso de lenguaje que hoy en día la sociedad utiliza para referirse a la discapacidad es percibido como discriminador. A su vez, se declara la importancia sobre la utilización de un lenguaje más inclusivo en la sociedad actual, señalando un concepto personal de discapacidad en base a las deficiencias y capacidades diferentes. Según las opiniones de los encuestados, el concepto personal de discapacidad se refiere a las deficiencias que las personas presentan en áreas motoras, sensoriales, mental, entre otros. Estas nociones hacen referencia a la percepción, la

Tabla 6. Lenguaje vinculado a discapacidad en estudiantes intervenidos.

\begin{tabular}{lcc}
\hline Uso de conceptos sobre discapacidad & Terminología no inclusiva & Terminología inclusiva \\
\hline Previo a intervención & $75 \%$ & $25 \%$ \\
Posterior a intervención & $33 \%$ & $67 \%$ \\
\hline
\end{tabular}

Fuente: Autores 
afectividad, la conciencia, la memoria y al lenguaje, como procesos mediados por un aprendizaje social, y que resignifican la visión del mundo (Botero, 2008, p. 17).

Eje temático II:

Visión sobre la discriminación hacia las personas con discapacidad

Se percibe que las formas más evidentes de discriminación y maltrato se vinculan con palabras o actitudes que tienen como consecuencia la humillación, agresión, valoración negativa o invisibilidad de las personas, la mayor parte de las veces avalados en el temor, desconocimiento, injusticia, falta de oportunidades, egoísmo e intolerancia.

A la gente solamente le importa ella misma (E2, p. 69);

La discriminación la aprendemos y la vivimos, yo creo que todos los días por A, B o C motivo (E4, p. 135);

yo creo que no es a propósito, yo creo que lo que se genera es una ceguera, o sea hasta que tú no te metes en el riel de la discapacidad la gente no la ve o la invisibiliza (E6, p. 16);

quizás la poca tolerancia, que tiene la gente, frente a este tipo de personas... y como repito de nuevo, hay que hacer una fuerte intervención en la gente para que cambie su pensamiento de una vez por todas, porque como está reaccionando la gente frente a la gente con discapacidad es casi inhumano (E2, p. 29).

Eje temático III:

Descripción del lenguaje personal y el utilizado por su comunidad educativa para referirse a personas con discapacidad

Los participantes en su totalidad hacen una diferencia en su lenguaje desde el conocimiento sobre la temática de discapacidad. Por lo tanto, antes de involucrarse con la discapacidad ellos refieren haber utilizado un lenguaje discriminador, y luego de interiorizarse en el tema, utilizan un lenguaje de tipo inclusivo.

\begin{abstract}
Haber antes de comenzar en los talleres... ya con responder la encuesta... yo pensé que estaba bien... pero la desinformación claramente... después de terminar los talleres... y ver las pocas que marqué con respecto a la primera... sinceramente me sentí... no sé... una muy mala persona, ya que sin darme cuenta, estaba tratando a gente con discapacidad... con palabras que no eran... y al momento de responder la encuesta y responderlas bien me sentí... alegre, por que sentí que aprendi cosas nuevas, que puedo hacer inclusión con la gente, para poder crear conciencia, y sobre todos ayudarme a mí mismo, para ayudar a la gente discapacitada (E2, p. 82);

Yo creo que yo utilizo o trato de utilizar un lenguaje que sea inclusivo... yo creo que el tema del lenguaje es bastante más que el lenguaje que se puede utilizar con una persona con discapacidad (E6, p. $50)$.
\end{abstract}

Tanto niñas, niños, jóvenes y docentes concuerdan que el lenguaje utilizado es inapropiado y despectivo, en las comunidades educativas, indicando que esto se debe a la desinformación y creencias erróneas.

Creo que en las comunidades educativas también caemos en eso, en no utilizar los conceptos adecuados, en discriminar, por lo tanto uno tiene que estar educando dentro y fuera de la comunidad educativa (E5, p. 45);

En general, se refieren de muy mala manera a ese tipo de personas, con lenguaje que no se debe, como... ejemplo, mira!, ahí va el ciego, ahí va el cojo, ahí va el sordo..., no sé... no sé... él está loco, cosa así (E2, p. 96).

Eje temático IV:

Respecto de la intervención realizada

Los participantes del estudio manifestaron un valioso avance en el tema de la inclusión al recibir educación pertinente. Ellos indicaron que 
utilizaban conceptos discriminatorios porque estos conceptos eran aprendidos sin cuestionamientos y eran socialmente compartidos y aceptados. Esta instancia permitió obtener aprendizajes que facilitaron la concientización y sensibilización en temáticas de discapacidad, marcando un precedente en este tipo de metodologías. Los entrevistados refieren que fue una instancia útil para el desarrollo personal, puesto que pudieron desarrollar habilidades sociales primordiales.

personalmente... muy buena... porque aparte de aprender cosas nuevas, palabras que quizá nunca hubiese pensado en escuchar... aprendí a ser más humano... y a tener en cierto modo tener más tolerancia con otro tipo de gente... con gente con discapacidad... y el ser más humano (E2, p. 106)

Durante el proceso vivido en los talleres educativos y luego exponer los resultados de sus propias investigaciones en un Congreso, fue percibida como novedosa y atractiva, desde un enfoque dinámico y participativo.

yo creo que fue potente ellos se vieron muy contentos y muy enganchados con la actividad, o sea, como muy afiatados creo que la debilidad es que el impacto en la comunidad no se vio en esta comunidad (E6, p. 96);

Desde el primer día el taller me pareció súper bueno, porque hicimos, ponte en su lugar..., y nos vendamos los ojos..., y pintamos con la boca... igual ahi sentí, ahí igual hubo un cambio porque sentíamos lo que sienten ellos... como que intercambiamos de puesto, de lugar (E1, p. 88);

Es el hecho de trasladarse y de estar en un Congreso, saber que están con otro grupo de pares que están ellos hay representado un proyecto, que insisto, puede que en este momento no tengan idea pero quizás no se po', en tres años más ellos van a leer algo y van a decir yo también estuve ahi en algún momento $y$ estuve participando con tal cosa (E6, p.
142).

De acuerdo a los aprendizajes obtenidos, expresan haber integrado nuevos conocimientos los cuales generaron motivación, concientización y cambios positivos en los participantes.

estos son los reales aprendizajes que uno tiene que ofrecerle a los niños y a las niñas, yo creo que cuando tú instalas estos temas cuando tú logras generar un cambio en ellos, cuando dejas una pequeña marquita vas convirtiéndolos en mejores personas, en mejores ciudadanos, personas más conscientes (E6, p. 123);

Sí, yo creo que hubo un impacto eh... y... yo creo que una cosa de conciencia, se hicieron más conscientes (E2, p. 145);

Conscientes, de ser más humanos entre ellos mismos, de ser más humanos con la gente que tiene otras capacidadesdiscapacidades (E5, p. 149);

Yo por lo menos veo en mis compañeros que han mejorado su lenguaje frente a ese tipo de personas..., han mejorado sus actitudes, como también yo... han sido más humanos, y eso se nota, se nota porque los ves más tolerantes (E2, p. 129).

La mayoría de los entrevistados refirió haber experimentado un cambio positivo en relación a la visión que tenían hacia las personas con discapacidad, indicando que este proceso les había ayudado a identificar situaciones de discriminación mediante el lenguaje, repercutiendo en una mejoría en las relaciones interpersonales entre sus propios compañeros y reconocimiento de habilidades sociales esenciales para el desarrollo personal.

Al finalizar la intervención, y concluidos los análisis de datos, los resultados de la investigación son entregados a cada grupo de trabajo, quienes presentan el material en el Congreso de Explora. Dicho material queda en sus respectivos establecimientos, con el compromiso de las autoridades de difundirlo en sus comunidades educativas. 


\section{CONCLUSIONES}

Los resultados de este estudio reflejan el impacto positivo de un proceso de sensibilización y capacitación dirigido a establecimientos educacionales sobre discapacidad y educación inclusiva en la ciudad de Punta Arenas. Su importancia radica en la generación de cambios actitudinales y comportamentales básicos al interior de las escuelas, lo que continúa siendo una necesidad en nuestra región y en nuestro país. La carencia de valores como la tolerancia, el respeto y la no discriminación de nuestra sociedad actual hace necesario que siempre se requieran mayores esfuerzos para continuar con este objetivo. Esta forma de discriminación radica en la escasa tolerancia a la diferencia, en la negación de la diversidad, siendo la educación la herramienta social llamada a capacitar a las generaciones para vivir, promover y reforzar la diversidad, y lamentablemente, no ha hecho sino que reproducir los mecanismos de la discriminación (Magendzo \& Donoso 2000, p. 6), elemento coincidente con las conclusiones de este estudio.

Los hallazgos indican que el lenguaje vinculado a discapacidad que utilizan los miembros de las comunidades educativas estudiadas es predominantemente un lenguaje discriminatorio y no inclusivo, lo que repercute en las relaciones interpersonales y convivencia escolar, vulnerando los derechos humanos de las personas con discapacidad, como lo reconocen numerosas investigaciones (Maturana, 1998; Echeverría, 1994; Van Dijk, 1996, 2003; Van Dijk \& Bixio, 2009; Magendzo \& Donoso, 2000, Barton, 2005, Fairclough, 2010). Esto es resultado de las propias concepciones atribuidas a discapacidad y discriminación por parte de actores estudiados, para quienes el concepto actual sobre discapacidad corresponde a una visión que no valida o incluso anula a estas personas como tales en la sociedad, considerándolas como algo extraño. Las causas identificadas son la adquisición y aprendizaje de este lenguaje sin cuestionamientos, su uso socialmente compartido y aceptado, la escasa educación recibida sobre el uso del lenguaje con enfoque en derechos humanos, la discriminación y sus repercusiones sociales. Estos resultados son coherentes con los estudios nacionales de Maturana
(1998), Magendzo y Donoso (2000), quienes hace más de una década vienen planteando la urgencia de incorporar estas temáticas valóricas en el ámbito educacional.

La percepción de los miembros de las comunidades educativas en relación a su evaluación del proceso de sensibilización y capacitación vivido resulta exitosa, puesto que reconocen haber integrado nuevos conocimientos, con la clara motivación de querer replicar y ampliar las actividades en su entorno escolar. Se reconoce un cambio positivo en relación a la visión sobre las personas con discapacidad, colaborando en la identificación de situaciones de discriminación mediante el lenguaje, repercutiendo en una mejoría en las relaciones interpersonales entre sus propios compañeros y reconocimiento de habilidades sociales importantes para el desarrollo personal. Aquí se pone de manifiesto la importancia que adquieren las formas de lenguaje que socialmente se utilizan para referirse a las personas que viven situaciones diversas, en particular sobre minorías sociales. Estas formas deben hacerse conscientes, en quienes educan a la comunidad, porque con las palabras se puede incluir, excluir, valorar o restar valor a quienes nos rodeen (INDH, 2012, p. 37).

Así también resulta primordial toda intervención educativa que contemple educación inclusiva y sobre derechos humanos en la educación chilena, con énfasis en la educación regional. Para la profesión de Terapia Ocupacional estas intervenciones son adoptadas con el fin de eliminar las situaciones de apartheid ocupacional y promoción de la justicia ocupacional en aquellos lugares donde exista discriminación en todas sus formas, inclusodesdelaremisión de barrerassociales, comenzando por las relaciones interpersonales y el uso de un lenguaje adecuado, en virtud de construir una sociedad más inclusiva para todos. El integrar y reconocer la igualdad de derechos de las personas con discapacidad requiere del reconocimiento y valoración de sus propias capacidades y cómo aportan a esta sociedad. Si el énfasis está puesto en su ser persona con una multidimensionalidad de características que las hacen únicas, y no sobre sus dificultades, puede lograr empoderarlos como sujetos plenos, rompiendo la relación vertical que genera el lenguaje actual mediante la discriminación y no valoración, transformando la discapacidad en 
diferencias personales y no en barreras sociales.

Desde una visión global, es posible aseverar que el unir estos esfuerzos con EXPLORA/ CONICYT, acercando la ciencia y la investigación a niños y niñas de la región, genera en ellos la valoración de los procesos investigativos, la identificación con los temas valóricos abordados y su compromiso por generar cambios en sus escuelas. En este sentido, resulta altamente valioso el apoyo que se puede ofrecer desde las Universidades al fomento de la investigación científica escolar, siempre y cuando se mantenga el foco centrado en la realidad e intereses de los niños y niñas, considerando su contexto y características socioeconómicas. Este tipo de instancias vuelve a demostrar las grandes brechas existentes en la educación de nuestra región y nuestro país, limitando las oportunidades de participación e involucramiento de niños y niñas pertenecientes a los establecimientos educativos más desfavorecidos.

A modo de síntesis, ésta fue una experiencia relevante, tanto para el desarrollo personal y académico de niños, niñas escolares y jóvenes universitarios, mediante la investigación se logró su acercamiento a una problemática existente en la realidad en la que viven y que generó un compromiso por querer promover cambios sociales y mejorar la calidad de vida de la población de Magallanes.

\section{LIMITACIONES DEL ESTUDIO Y PROSPECTIVA}

La experiencia obtenida permite concluir que es posible mejorar mucho más ciertos elementos del proceso. Como limitaciones de la investigación, se puede señalar que resulta restrictivo el impacto de intervenciones breves en el tiempo y que no cuenten con una mirada multidisciplinaria. Apuntando en este sentido, es posible indicar que es conveniente seguir investigando y desarrollando una aproximación más global al fenómeno de la lingüística y sus implicancias sociales y educativas con enfoque en derechos humanos, que cuente con la participación de la multiplicidad de actores del proceso educativo. Asimismo, se debe destacar la urgente necesidad de avanzar hacia la conformación de una convivencia escolar y sociocultural inclusiva, en todos los niveles educativos hasta llegar a la educación superior. Para ello, sería conveniente profundizar en buenas prácticas de convivencia escolar, en su real presencia en las bases curriculares de la educación nacional y en los mecanismos de acreditación que supervisen el proceso. Y por supuesto, debe instalarse fuertemente en la formación inicial docente, donde bajo estas barreras culturales subyacen supuestos sobre la diversidad o diferencia como problema y no como recurso potenciable (Lissi et al. 2011).

Igualmente, se debe enfatizar que los beneficios de cualquier tipo de intervención en esta área son limitados si las políticas educativas no priorizan la temática a nivel nacional, y si quienes lideran los contextos educativos no promueven en forma sistemática el desarrollo de una cultura inclusiva en sus comunidades.

Por último, en estos procesos educativos y de sensibilización, es importante realzar el rol imprescindible de la Universidad de Magallanes como única institución pública de educación superior en la región, cuya responsabilidad social es la promoción de cambios sociales y la inclusión social de grupos vulnerables. Su impacto en la educación y cultura de la región, incluso del país, mediante el trabajo de los profesionales que ella forma son incalculables.

\section{BIBLIOGRAFÍA}

Amemiya, I., Oliveros, M., \& Barrientos, A. (2009). Factores de riesgo de violencia escolar (bullying) severa en colegios privados de tres zonas de la sierra del Perú. Anales de la Facultad de Medicina, 70(4), 255-258.

Barton, L. (2005). Discapacidad y sociedad. Madrid: Morata

Blanco, R. (2004). Temario abierto sobre educación inclusiva. Santiago: UNESCO.

Botero, P. (2008). Representaciones y Ciencias sociales; Una perspectiva epistemológica y metodológica. Buenos Aires: Espacio

Carter, B. B., \& Spencer, V. G. (2006). The fear factor: Bullying and students with disabilities. International Journal of Special Education, 21(1), 11-23.

Cifuentes, R. (2011). Diseño de proyectos de investigación cualitativa. Buenos Aires: Noveduc.

Comisión Nacional de Verdad y Reconciliación (1996). Informe de la Comisión Nacional de la Verdad y Reconciliación. Santiago, Chile: Corporación Nacional de Reparación y Reconciliación. Recuperado de http://www.ddhh. gov.cl/ddhh_rettig.html 
Díaz-Aguado, M., Martínez, R., \& Martín, J. (2013). El acoso entre adolescentes en España. Prevalencia, papeles adoptados por todo el grupo y características a las que atribuyen la victimización. Revista de Educación, (362), 348-379.

Duncan, R. (1999). Maltreatment by parents and peers: The relationship between child abuse, bully victimization and psychological distress. Child Maltreatment, 4(1), 45-55.

Echeverría, R. (1994). Ontología del lenguaje. Santiago: Dolmen Ediciones.

Elliot, J. (1996). El cambio educativo desde la investigaciónacción. Madrid: Morata.

Fairclough, N. (2010). Language and power. Harlow: Longman.

FONADIS/INE (2004). Primer Estudio Nacional de la Discapacidad en Chile ENDISC. Recuperado de http:// www.senadis.gob.cl/documentos/endisc

Glaser, B. G., \& Strauss, A. L. (1967). The discovery of grounded theory: Strategies for qualitative research. Chicago: Aldine Pub. Co.

Instituto Nacional De Derechos Humanos (2012). Ideas para introducir los Derechos Humanos en el Aula. Santiago: Autor.

Islas, H. (2005). Lenguaje y discriminación. Cuadernos de la igualdad. México D.F.: Consejo Nacional para Prevenir la Discriminación.

Krause, M. (2002). Investigación-acción participativa: Una metodología para el desarrollo de autoayuda, participación y empoderamiento. En J. Durston \& F. Miranda (Eds.), Experiencias y metodología en la investigación participativa (pp. 41-67). Santiago, Chile: CEPAL - Serie Políticas Sociales.

Lissi, M. R., Grau, V., Salinas, M., \& Sebastian, C. (2011). Avance, desafíos y propuestas en relación a la Educación Inclusiva. Simposio presentado en el XXXIII Congreso Interamericano de Psicología, Medellín, 26-30 junio. MS.

Magendzo, A., \& Donoso, P. (Eds.). (2000). Cuando a uno lo molestan... Un acercamiento a la discriminación en la escuela. LOM Ediciones y PIIE, Santiago.

Maturana, H. (1998). Emociones y lenguaje en educación y política. Belo Horizonte: UFMG.

MIDEPLAN (2010). Ley Num. 20.422: Establece Normas Sobre Igualdad de Oportunidades e Inclusión Social de Personas con Discapacidad. Biblioteca del Congreso Nacional de Chile, Santiago.

Milicic, N., \& López, S. (2008). Hostigamiento escolar: Propuestas para la elaboración de políticas públicas. Pontificia Universidad Católica de Chile, Vicerrectoría de Comunicaciones y Dirección de Asuntos Públicos abril $\mathrm{N}^{\circ} 15$, Santiago.

Miller, T., Beane, A., \& Kraus, R. (1998). Clinical and cultural issues in diagnosing and treating child victims of peer abuse. Child Psychiatry and Human Development, 29(1), 21-32.

MINEDUC \& UNESCO. (2005). Primer Estudio Nacional de Convivencia Escolar. Recuperado de http://www.comisionunesco.cl

MINEDUC (2005). Política Nacional de Educación Especial: Nuestro compromiso con la diversidad. Recuperado de http://web.integra.cl/doctos_cedoc/archivos/documentos/NUESTRO_COMPROMISO_CON_LA_DIVERSIDAD.pdf

MINEDUC (2011). Encuesta Nacional de prevención, agresión y acoso escolar. $8^{\circ}$ básico, SIMCE 2011. Recuperado de http://www.mineduc.cl

MINEDUC (2011). Ley Num. 20.536: sobre violencia escolar. Biblioteca del Congreso Nacional de Chile, Santiago.

MINEDUC (2012). Orientaciones técnicas para programas de integración escolar (PIE). Recuperado de http://www. educacionespecial.mineduc.cl/usuarios/edu.especial/ File/2012/SEPTIEMBRE2012/OrientTecPIEWEB. PDF

Mishna, F. (2003). Learning disabilities and bullying double jeopardy. Journal of Learning Disabilities, 36(4), 336-347.

ONU (1948). Declaración Universal de los Derechos $\mathrm{Hu}$ manos. Recuperado de http://www.un.org/es/documents/udhr/

ONU (2007). Convención sobre los derechos de las personas con discapacidad y Protocolo Facultativo. Recuperado de http://www.un.org/disabilities/documents/convention/convoptprot-s.pdf

Organización De Estados Americanos (1999). Convención Interamericana para la Eliminación de Todas las Formas de Discriminación Contra las Personas con Discapacidad. Recuperado de http://www.oas.org/ juridico/spanish/tratados/a-65.html

Organización Mundial De La Salud (2001). Clasificación Internacional del funcionamiento de la discapacidad y de la salud, versión abreviada. Recuperado de http:// www.who.int/iris/handle/10665/43360\#sthash. mlEz1Nbe.dpuf

Rose, C. A., Monda-Amaya, L. E., \& Espelage, D. L. (2011). Bullying perpetration and victimization in special education: A review of the literatura. Remedial and Special Education, 32(2), 114-130.

SENADIS (2010). Recomendaciones para el uso correcto del 
Lenguaje en temas relacionados con Discapacidad. Recuperado de http://www.senadis.gob.cl/centro/ grafico.php

Strauss, A., \& Corbin, J. (2002). Bases de la investigación cualitativa. Técnicas y procedimientos para desarrollar la teoría fundamentada. Medellín: Universidad de Antioquía.

Townsed, E., \& Whiteford, G. (2007). Una estructura de participación en el marco de la justicia ocupacional: Procesos prácticos basados en la comunidad. En F. Kronenberg, S. Simó \& N. Pollard (Eds), Terapia Ocupacional Sin Fronteras: Aprendiendo del Espíritu de Supervivientes, (pp.
110-126). Buenos Aires: Médica Panamericana.

UNICEF - Fondo de las Naciones Unidas para la Infancia (2004). Convivencia en el ámbito escolar. Informe cuantitativo primera parte. Recuperado de http:// www.unicef.cl

Van Dijk, T. A. (2003). Dominación étnica y racismo discursivo en España y América Latina. Barcelona: Gedisa.

Van Dijk, T. A., \& Bixio, A. (2009). Discurso y poder: Contribuciones a los estudios críticos del discurso. Barcelona: Gedisa.

Van Dijk, T. A. (1996). Seminario sobre análisis crítico del discurso, Instituto Caro y Cuervo, Santafé de Bogotá, MS. 